\title{
The Vibrational Spectra of Ethylene Trithiocarbonate and Ethylene Thiourea
}

\author{
PETER KLFBOE
}

Department of Chemistry, University of Oslo, Oslo 3, Norway

\begin{abstract}
The infrared spectra of two cyclic thioketones, ethylene trithiocarbonate and ethylene thiourea, were recorded in the region $4000-$ $220 \mathrm{~cm}^{-1}$ as solids. Raman spectra of both compounds in the solid state were obtained with $\mathrm{He}-\mathrm{Ne}$ laser excitation. Ethylene trithiocarbonate is soluble in organic solvents and infrared and Raman spectra of this compound were recorded in solution and semiquantitative polarization data presented.

The vibrational spectra of both molecules suggest non-planarity of the 5-membered rings and the molecular symmetry is not higher than $C_{s}$ or $C_{2}$. A tentative assignment of the vibrational fundamentals for both molecules are presented. The complex formation between these molecules and iodine have been studied qualitatively.
\end{abstract}

The spectral data of very few five-membered heterocyclic rings have been 1 reported. These molecules are of special interest regarding their stereochemistry and it is of importance to determine if they are planar in the various states of aggregation. In a planar configuration they have $C_{2 v}$ symmetry, which should be evident from the vibrational spectra since the vibrations of species $A_{2}$ should be Raman active, but infrared inactive.

The infrared spectra of ethylene trithiocarbonate (ETC) and ethylene thiourea (ETU) have been reported by Mecke and co-workers. ${ }^{1}$ However, they obtained only a very incomplete Raman spectrum of ETU and no spectrum of ETC. This was undoubtedly caused by the strong yellow colour of the latter which completely prevents the use of the $4358 \AA \mathrm{Hg}$-line for Raman excitation. The infrared and Raman spectra in the high frequency region of the related ethylene carbonate have been reported and a partial interpretation of the spectra presented..$^{2}$ The vibrational spectra of the five-membered heterocyclic ring tetramethylene sulphone is interpreted on the basis of nonplanarity. ${ }^{3}$ Data to be presented for tetramethylene sulphoxide ${ }^{4}$ suggest a puckered ring also for this compound.

Various papers have dealt with the vibrational spectra of thiourea and its complexes with metal halides ${ }^{5,6}$ and hydrogen chloride. ${ }^{7}$ Solvent effect 
studies of the thiocarbonyl group have been reported ${ }^{8}$ and the group frequency of the $\mathrm{C}=\mathrm{S}$ group found to be strongly sensitive to neighbouring $\mathbf{N}$-atoms. ${ }^{9-10}$ $\mathrm{X}$-Ray crystallographic studies of ETU ${ }^{11}$ and ethylene carbonate ${ }^{12}$ reveal that these molecules deviate from planarity in the solid state.

Since a Raman spectrometer with laser excitation was available for some time, we decided to record the infrared and Raman spectra of ETC and ETU. A tentative assignment of the fundamental frequencies for both molecules is reported, but because of the large number of atoms and low symmetry of these compounds it should be considered as tentative.

\section{EXPERIMENTAL}

The two compounds studied were commercial samples from the $\mathrm{K} \& \mathrm{~K}$ laboratories (ETC) and the Eastman company (ETU). They were both purified by fractional recrystallization from alcohol. The solvents were of spectroscopic quality (Merck, uvasole) and they were not purified further.

The infrared spectra were recorded with Beckman model IR-9 (4000-400 $\left.\mathrm{cm}^{-1}\right)$ and Perkin-Elmer model $621\left(4000-220 \mathrm{~cm}^{-1}\right)$ spectrometers, calibrated with $\mathrm{NH}_{3}$, $\mathrm{CH}_{4}$, and atomospheric $\mathrm{H}_{2} \mathrm{O}$ and $\mathrm{CO}_{2}$. The solid samples were recorded as $\mathrm{KBr}$-tablets and as mulls in nujol and fluorolube. ETC is soluble in organic solvents and was dissolved in $\mathrm{CCl}_{4}, \mathrm{CS}_{2}$, and $\mathrm{CHCl}_{3}$. ETU on the other hand is only slightly soluble in practical solvents and only the most intense infrared bands could be detected in solution.

Table 1. Infrared spectral data for ethylene trithiocarbonate in solution (in $\mathrm{cm}^{-1}$ ).

\begin{tabular}{|c|c|c|c|c|c|c|c|}
\hline \multicolumn{4}{|c|}{ Solvent } & \multicolumn{4}{|c|}{ Solvent } \\
\hline $\begin{array}{l}3920 \\
3815 \\
3661 \\
3440 \\
2986 \\
2930 \\
2860 \\
2836 \\
2714 \\
2361 \\
2295 \\
2260 \\
2132 \\
2086 \\
1950 \\
1761 \\
1711 \\
1606 \\
1576 \\
1422 \\
1383 \\
1308 \\
1280 \\
1251\end{array}$ & $\begin{array}{l}\text { w, }{ }^{a} \text { bd } \\
\text { w } \\
\text { w } \\
\text { vw } \\
\text { s } \\
\text { s } \\
\text { w } \\
\text { m } \\
\text { vw } \\
\text { w } \\
\text { vw } \\
\text { m } \\
\text { m } \\
\text { vw } \\
\text { w } \\
\text { m } \\
\text { w } \\
\text { vw } \\
\text { w } \\
\text { s } \\
\text { vw } \\
\text { vw } \\
\text { vs, sp } \\
\text { s, sp }\end{array}$ & $\begin{array}{l}1422 \\
1384 \\
1311 \\
1286 \\
1254\end{array}$ & $\begin{array}{l}\text { s } \\
\text { vw } \\
\text { vw } \\
\text { vs, sp } \\
\text { s, sp }\end{array}$ & $\begin{array}{r}1152 \\
1106 \\
\text { ca. } 1063 \\
\\
\\
1008 \\
984 \\
956 \\
946 \\
930 \\
880 \\
831 \\
\\
668 \\
\\
583 \\
501 \\
475 \\
453 \\
381 \\
246\end{array}$ & $\begin{array}{l}\text { s } \\
\text { w, sd } \\
\text { vvs } \\
\text { m } \\
\text { s } \\
\text { s, sd } \\
\text { s } \\
\text { w } \\
\text { vs } \\
\text { vs } \\
\text { vs, sp } \\
\text { vw } \\
\text { s } \\
\text { s } \\
\text { s } \\
\text { s } \\
\text { m? }\end{array}$ & $\begin{array}{r}1156 \\
1112 \\
1075 \\
1054 \\
1040 \\
1010 \\
988 \\
958 \\
951 \\
935 \\
886 \\
836 \\
750 \\
677 \\
657 \\
579 \\
502 \\
476 \\
453 \\
382\end{array}$ & $\begin{array}{l}\text { s } \\
\text { w, sd } \\
\text { vvs } \\
\text { w, sd } \\
\text { w } \\
\text { m } \\
\text { s } \\
\text { s, sd } \\
\text { s } \\
\text { w } \\
\text { vs } \\
\text { vs } \\
\text { m } \\
\text { vs, sp } \\
\text { w } \\
\text { vw, bd } \\
\text { s } \\
\text { s } \\
\text { s } \\
\text { s }\end{array}$ \\
\hline
\end{tabular}

$a$ The following abbreviations have been used: $w$, weak; $\mathrm{m}$, medium; s, strong; $\mathrm{v}$, very; $\mathrm{sp}$, - sharp and bd, broad. 
We tried to record the infrared spectra of ETC in the vapour phase by means of a $10 \mathrm{~m}$ folded cell and by a $9 \mathrm{~cm}$ cell which was heated to $c a .100^{\circ} \mathrm{C}$. However, not even the strongest absorption bands could be detected in any of the spectra.

Mixed solutions of ETC- $\mathrm{I}_{2}$ and ETC-ICl in $\mathrm{CS}_{2}$ were prepared and scanned immediately. The complexes exhibited a number of new infrared bands compared to ETC itself. Negligible spectral changes occurred when the ETC $-I_{2}$ solution was rescanned after $1 \mathrm{~h}$. In the ETC-ICl solution, however, the spectrum changed with time, probably because of irreversible oxidation or substitution reactions.

The Raman spectra were recorded with a Cary 81 Raman spectrometer equipped with a Spectra Physics model $125,50 \mathrm{MW} \mathrm{He}-\mathrm{Ne}$ laser, using the $6328 \AA$ radiation. The solutions were filled in capillary tubes of silica, approximately $0.7 \mathrm{~mm}$ in diameter and $5 \mathrm{~cm}$ long, containing approximately $20 \mu \mathrm{l}$. The capillary was "sealed" with glycerol, which was also used between the sample tube and the spectrometer lens to achieve good optical contact. Solid pellets of pure ETC and ETU were prepared in a micro die, wetted with glycerol and placed in contact with the spectrometer lens.

Table 2. Raman spectral data for ethylene trithiocarbonate (in $\mathrm{cm}^{-1}$ ).

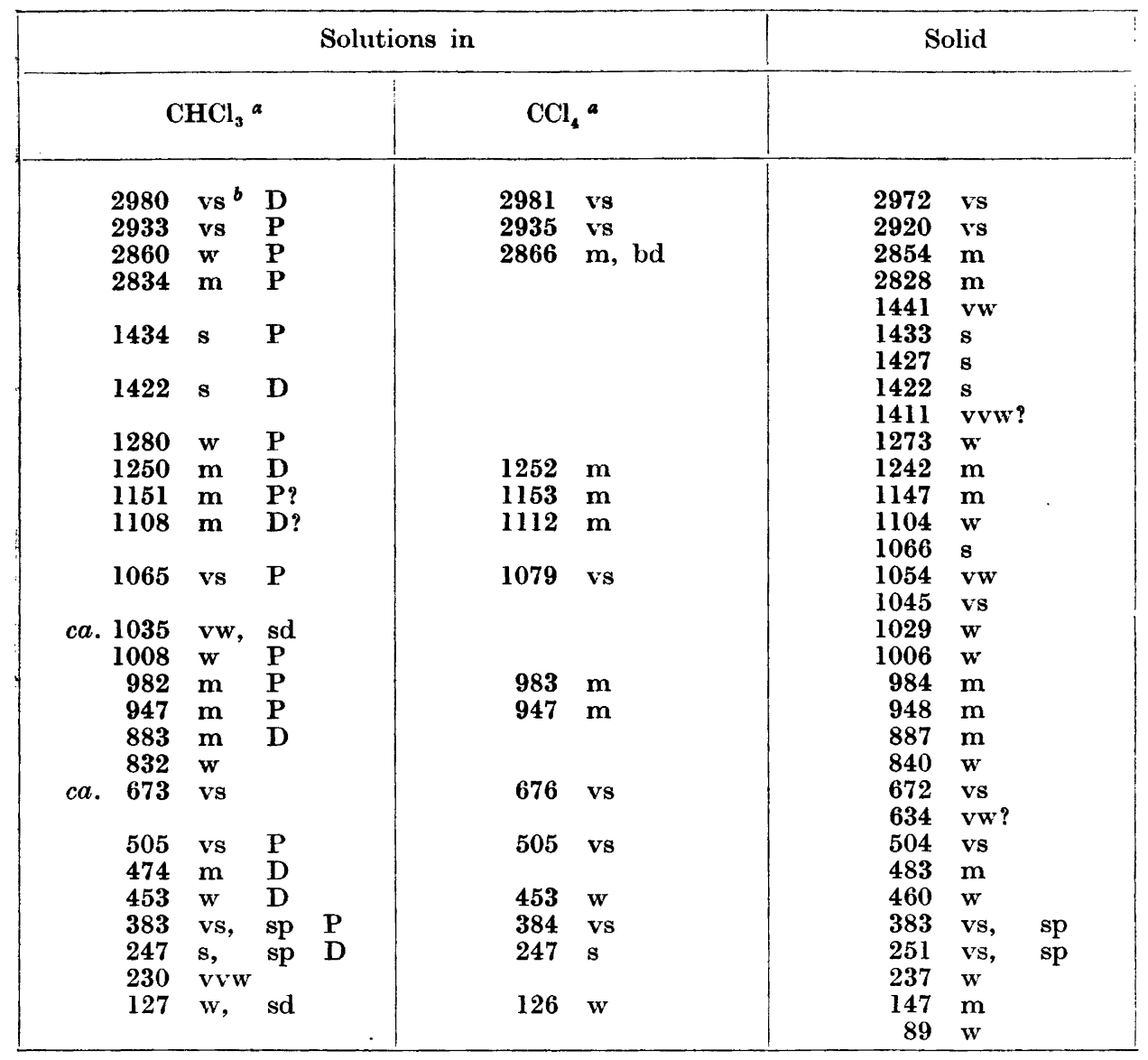

a The solutions were saturated.

$b$ For abbreviations, see Table 1 . 


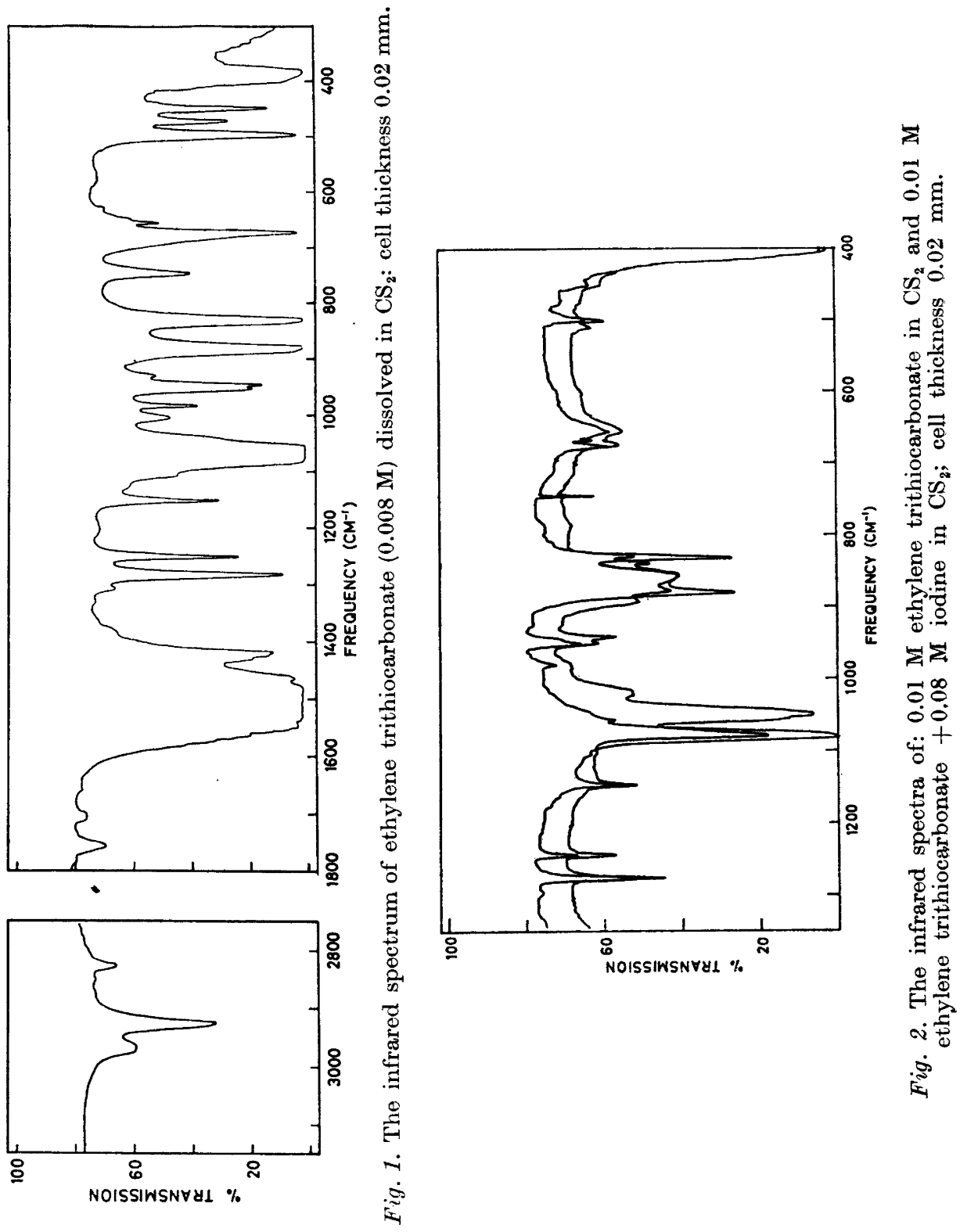

Acta Chem. Scand. 22 (1968) No. 5 
An attempt was made to record the Raman spectrum of a mixed ETC $-\mathrm{I}_{2}$ solution in $\mathrm{CHCl}_{3}$. One of the shifted ETC bands was detected, although this technique is very difficult with the Raman effect. ${ }^{13}$

Semiquantitative polarization measurements were carried out, using a half wave plate between the laser and the sample tube. A polarizer which can be rotated $90^{\circ}$ was

Table 3. Vibrational spectral data of solid ethylene thiourea and tentative fundamental assignments.

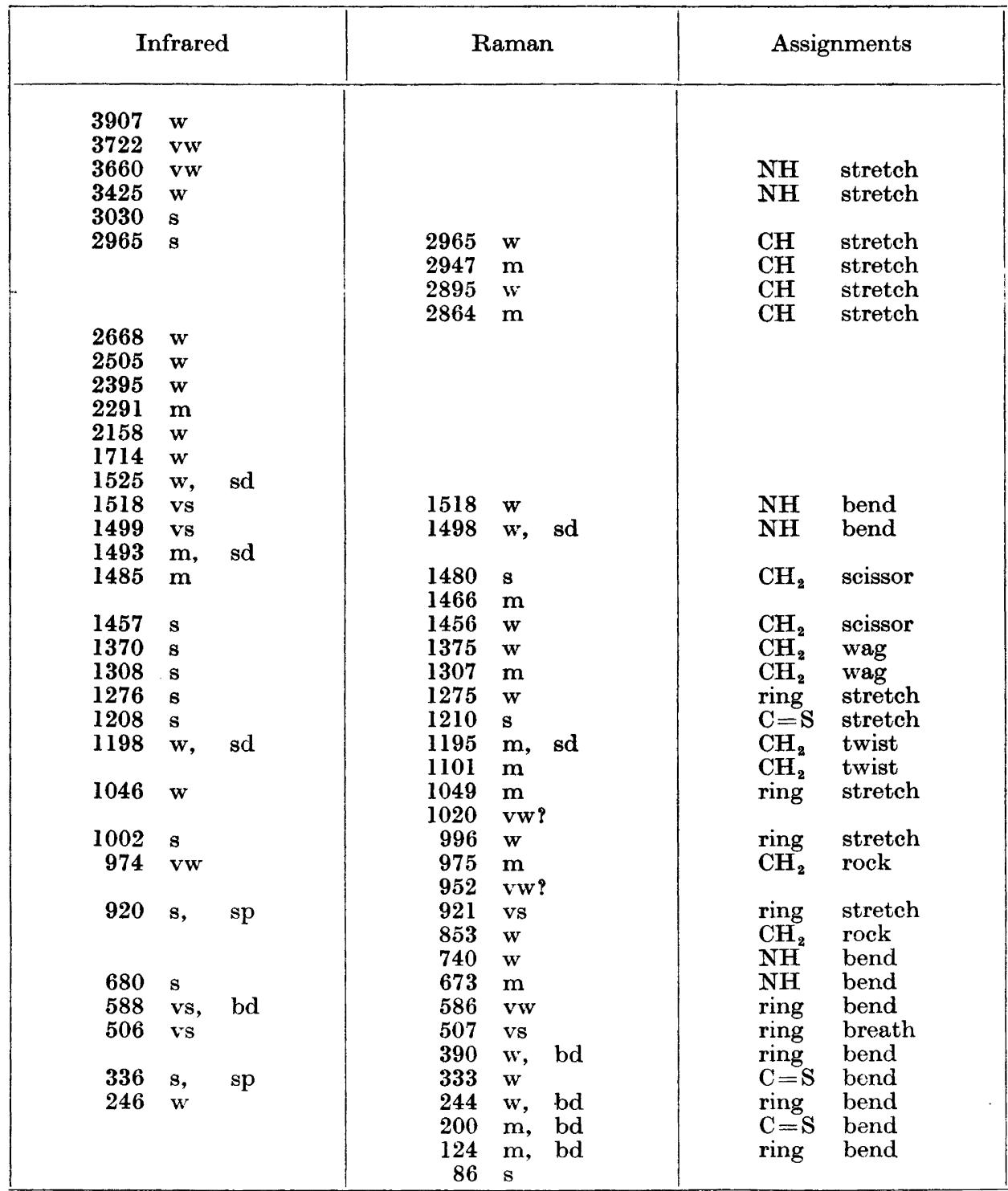

${ }^{a}$ For abbreviations see Table 1. 
placed between the sample tube and the spectrometer entrance slit. No polarization data could be obtained for ETU which was recorded as a solid pellet only.

A qualitative Raman spectrum of ETC dissolved in $\mathrm{CCl}_{4}$ was recorded, using the green $\mathrm{Hg}$ line at $5461 \AA$ in a conventional Cary 81 spectrometer with Toronto arc. This spectrum was very poor compared to the one obtained with laser excitation and wide spectral regions were covered by other $\mathrm{Hg}$ lines.

The infrared spectra of ETC in $\mathrm{CS}_{2}$ solution is shown in Fig. 1 and the spectra of the ETC $-I_{2}$ solutions are shown in Fig. 2. The Raman spectra of ETC are shown in Fig. 3 and the observed infrared and Raman frequencies are listed in Tables 1 and 2. For ETU the infrared and Raman frequencies are listed in Table 3.

\section{RESULTS AND DISCUSSION}

Ethylene trithiocarbonate

No structural determination has been reported for ETC and the symmetry of this molecule is therefore uncertain. The related ethylene carbonate is reported to be non-planar in the crystal ${ }^{12}$ but it has been tentatively considered planar in solution. ${ }^{2}$ A planar molecule of ETC should have $C_{2 v}$ symmetry, non-planar models $C_{2}, C_{s}$ or $C_{1}$ symmetry. The activities of the 24 normal vibrations for the appropriate point groups are listed in Table 4. The most

Table 4. Classification of the normal modes in ethylene trithiocarbonate for various possible point groups.

\begin{tabular}{|c|c|c|c|c|c|c|c|c|c|c|c|}
\hline \multicolumn{3}{|c|}{$C_{2 v}$} & \multicolumn{3}{|c|}{$C_{s}$} & \multicolumn{3}{|c|}{$C_{2}$} & \multicolumn{3}{|c|}{$C_{1}$} \\
\hline Species & IR & $\mathrm{Ra}$ & Species & IR & $\mathbf{R a}$ & Species & IR & $\mathbf{R a}$ & Species & IR & $\mathbf{R a}$ \\
\hline $\begin{array}{ll}8 & A_{1} \\
4 & A_{2} \\
7 & B_{1} \\
5 & B_{2}\end{array}$ & $\begin{array}{r}\mathbf{a} \\
\mathbf{i a} \\
\mathbf{a} \\
\mathbf{a}\end{array}$ & $\begin{array}{l}\mathbf{P} \\
\mathbf{D} \\
\mathbf{D} \\
\mathbf{D}\end{array}$ & $\begin{array}{l}13 A^{\prime} \\
11 A^{\prime \prime}\end{array}$ & $\begin{array}{l}\mathbf{a} \\
\mathbf{a}\end{array}$ & $\begin{array}{l}\mathbf{P} \\
\mathbf{D}\end{array}$ & $\begin{array}{l}12 \mathrm{~A} \\
12 \mathrm{~B}\end{array}$ & $\begin{array}{l}a \\
a\end{array}$ & $\begin{array}{l}\mathbf{P} \\
\mathbf{D}\end{array}$ & $24 \mathrm{~A}$ & $\mathbf{a}$ & $\mathbf{P}$ \\
\hline
\end{tabular}

The following abbreviations have been used: a, active; ia, inactive; $\mathbf{P}$, Polarized and $\mathrm{D}$, depolarized.

prominent spectral feature in assigning the right point group would be the fact that vibrations belonging to species $A_{2}$ are infrared inactive, but Raman active and such non-coincidences should be fairly easy to pick out. To distinguish between the point groups $C_{2}, C_{s}$, or $C_{1}$ is hardly possible in this case since it is a well known fact that depolarized Raman lines are very difficult to determine. Band-contours of infrared vapour spectra can in many cases give very useful information about the symmetry, but the vapour pressure of ETC was much too low.

It appears from Tables 1 and 2 that no clear-cut cases are observed in which Raman bands have no infrared counterparts. This observation cannot be considered absolutely conclusive since inactive infrared vibrations might show up in solution because of breaking-down of the selection rules. However,

Acta. Chem. Scand. 22 (1968) No. 5 
it seems justified to conclude that ETC is non-planar in the solid as well as in solution. The number of Raman bands which appear polarized, suggest that the point group should be $C_{2}$ (two-fold axis) or $C_{s}$ (symmetry plane).

Vibrational assignments. Exactly four bands are observed in the infrared and the Raman spectra around $3000 \mathrm{~cm}^{-1}$ and they are assigned to the four $\mathrm{C}-\mathrm{H}$ stretching modes. If ETC had $C_{2 v}$ symmetry, one of these modes should be of species $A_{2}$. However, all the four Raman bands have infrared counterparts, and the very weak infrared band at $2857 \mathrm{~cm}^{-1}$ cannot be an $A_{2}$ fundamental since the corresponding Raman band is definitely polarized.

The remaining fundamental modes are all expected below $1500 \mathrm{~cm}^{-1}$. It seems significant that approximately 20 certain Raman bands which all have infrared counterparts are observed in this region and it seems reasonable to assign these bands to the remaining 20 fundamentals. The bands in the region $1450-1100 \mathrm{~cm}^{-1}$ of which three are polarized and three seem depolarized have been assigned to the six $\mathrm{CH}_{2}$ deformation modes: scissoring, wagging and twisting. With $C_{2}$ or $C_{s}$ symmetry, one vibration of each type should be symmetrical, the other asymmetrical in agreement with the polarization data. The $1065 \mathrm{~cm}^{-1}$ band is very intense in infrared and Raman, it is affected by solvent polarity and state of aggregation and is undoubtedly connected with the $\mathrm{C}=\mathrm{S}$ stretching mode. The two methyl rocking modes are somewhat

Table 5. Tentative vibrational fundamentals in ethylene trithiocarbonate.

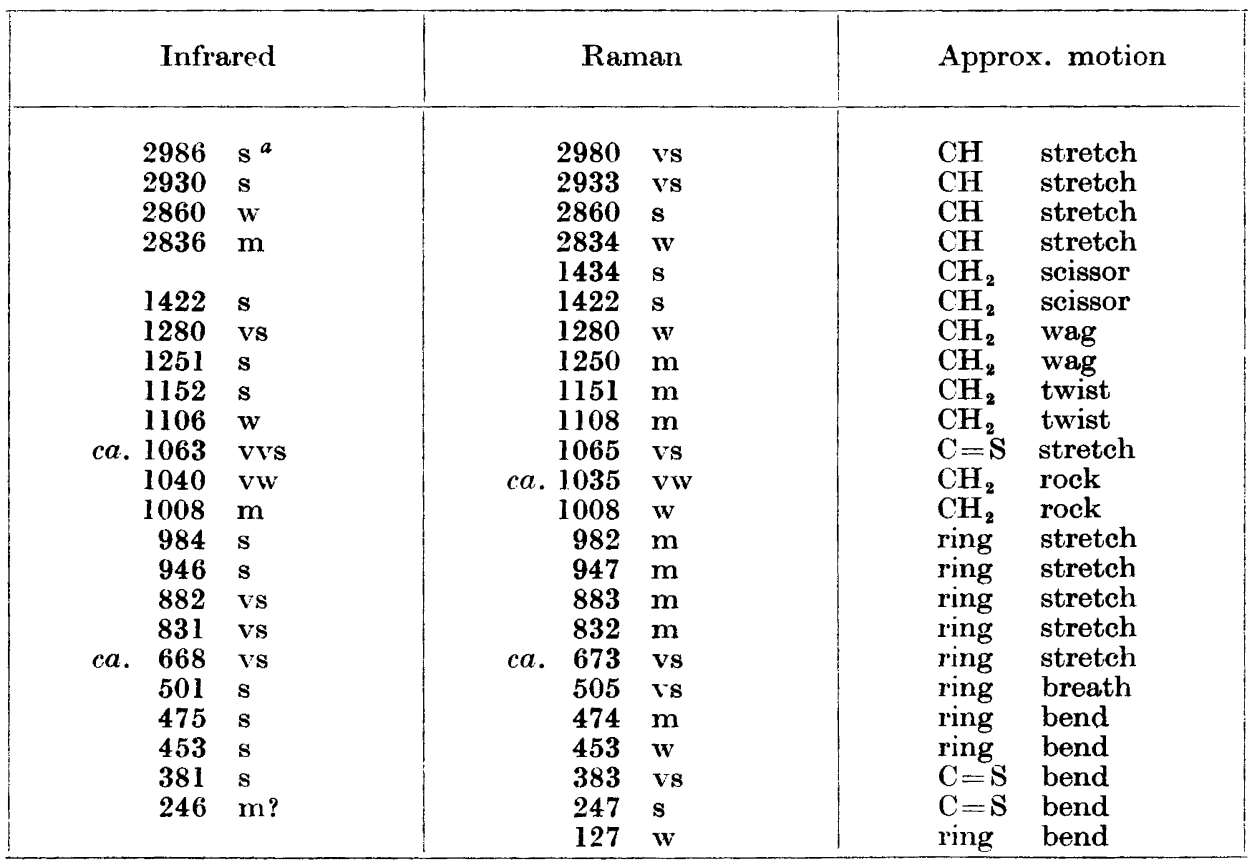

a For abbreviations see Table 1 . 
arbitrarily assigned to the bands at 1035 and $1008 \mathrm{~cm}^{-1}$, which both are weak to medium intense in infrared and Raman.

The remaining 11 fundamentals are all connected with the ring stretching, bending and torsional modes for which the group frequency concepts are of no use. Moreover, there are very few other aliphatic 5-membered rings with heteroatoms which have been thoroughly studied spectroscopically. In the 5-membered, planar heteroaromatic rings as, e.g., thiophene, ${ }^{14}$ pyrrole ${ }^{15}$ or the oxadiazoles ${ }^{16}$ no fundamental frequency is reported below $600 \mathrm{~cm}^{-1}$. In ETC, six bands believed to be fundamentals are observed below $600 \mathrm{~cm}^{-1}$, indicating that this molecule is "soft" and flexible with much lower ring bending and stretching force constants. No real distinction can be made between most of the skeletal stretching and deformation modes, except for a planar ring in which the $A^{\prime \prime}$ modes are pure bending vibrations. The bands in the region $1000-700 \mathrm{~cm}^{-1}$ are assumed to involve mainly stretching vibrations, with the strong, polarized Raman band at $505 \mathrm{~cm}^{-1}$ assigned to the ring "breathing" mode. The "in plane" and "out of plane" $\mathrm{C}=\mathrm{S}$ bending modes are assigned to the bands at 383 and $247 \mathrm{~cm}^{-1}$, respectively. The "envelope" bending mode around the S-S line should be the lowest fundamental, observed at $127 \mathrm{~cm}^{-1}$.

The remaining infrared bands can be interpreted as binary combinations or overtones. Practically all the infrared bands below $1500 \mathrm{~cm}^{-1}$ are assigned to fundamentals, exceptions are those at 956 and $750 \mathrm{~cm}^{-1}$ which can be explained as $453+505=958$ and $247+505=752$, respectively.

Complex formation. ETC contains a thiocarbonyl and two sulphide groups which are all potentially strong donor sites for complex formation to iodine. Dissolved in $\mathrm{CCl}_{4}$ ETC has electronic absorption bands at 456, 314, and 294 $\mathrm{m} \mu$. The visible absorption band of iodine dissolved in $\mathrm{CCl}_{4}$ at $517 \mathrm{~m} \mu$ is blue shifted to $469 \mathrm{~m} \mu$ on complex formation with ETC, suggesting an intermediately strong interaction. ${ }^{17}$ Solutions with a constant iodine concentration and varying ETC concentrations pass through an isosbestic point at $497 \mathrm{~m} \mu$, suggesting a 1:1 complex. Because of the strong ETC absorption in the ultraviolet region we were not able to observe any charge transfer band, characteristic of the ETC $-\mathrm{I}_{2}$ complex. The data: $K c\left(25^{\circ}\right)=451 \mathrm{~mole}^{-1}$ and $-\Delta H^{\circ}=8.5 \mathrm{kcal} \mathrm{mole}^{-1}$ are reported for the ETC- $\mathrm{I}_{2}$ complex. ${ }^{18}$

The ETC- $\mathrm{I}_{2}$ complex was studied in $\mathrm{CS}_{2}$ and $\mathrm{CCl}_{4}$ solution in the infrared region. Solutions in $\mathrm{CS}_{2}$ containing the same amount of ETC and varying iodine concentrations are shown in Fig. 2. It appears that in agreement with our earlier observations on iodine complexes, ${ }^{19}$ the spectral changes can be classified as displaced bands and enhanced bands. Most significant is the "red shift" observed for the $C=S$ stretching band at $1071 \mathrm{~cm}^{-1}$, revealing that the complex formation occurs from the thiocarbonyl sulphur. The shifted and enhanced bands are listed in Table 6. It appears that certain bands are "blue shifted" on complex formation. The $\mathrm{C}=\mathrm{S}$ bending and some of the skeletal bending modes are expected to be "blue shifted", but these effects are not very easy to predict. A further complex formation of $1: 2$ or even $1: 3$ stoichiometry cannot be excluded under these conditions of high iodine concentrations.

Perturbations of the I-I stretching mode on complex formation to ETC have been reported. ${ }^{13}$ An attempt was made to study perturbations in the Raman spectrum of ETC on complexing with iodine. In a 1 M solution of

Acta Chem. Scand. 22 (1968) No. 5 


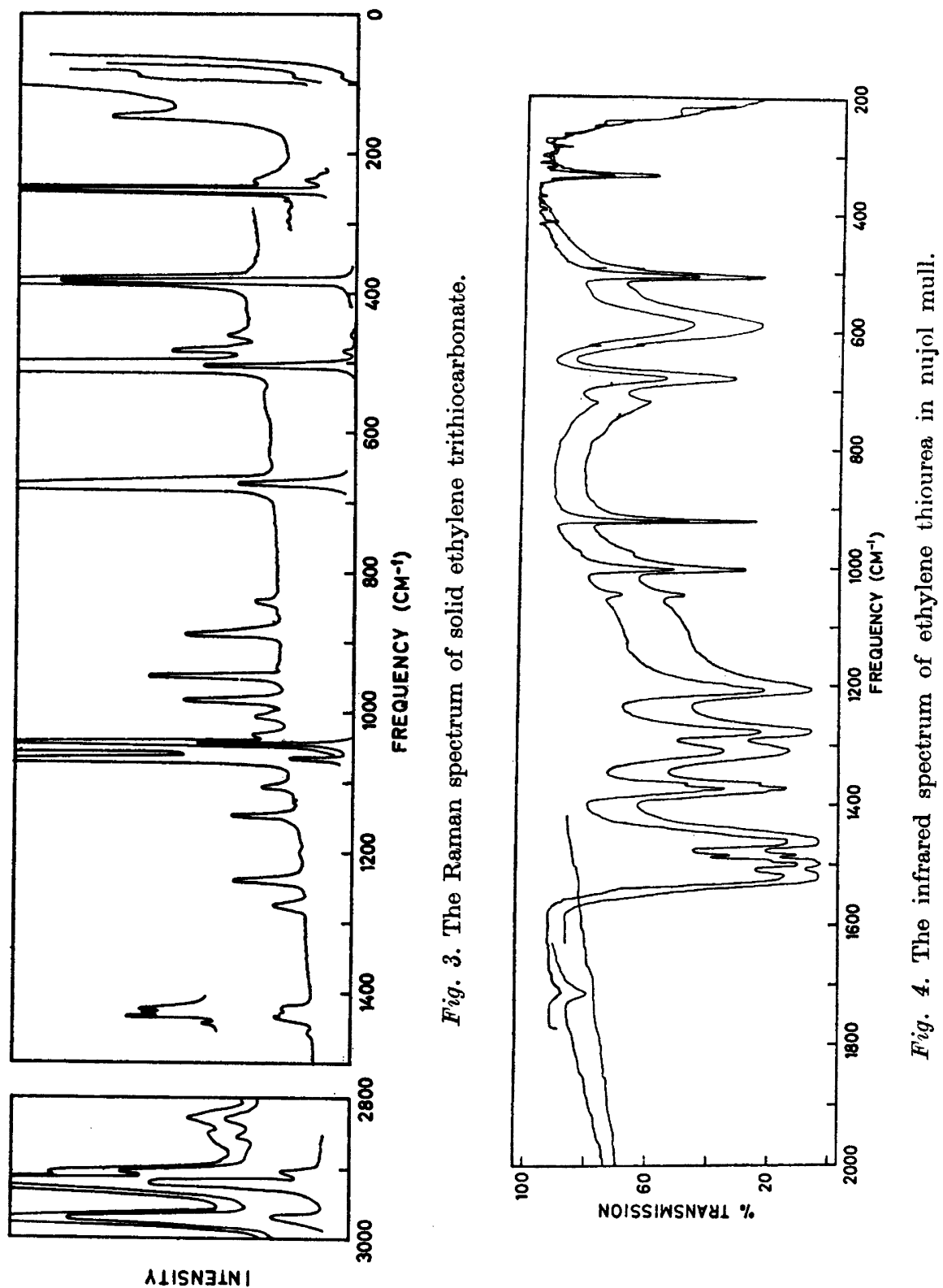

Acta Chem. Scand. 22 (1968) No. 5 
Table 6. Infrared spectral shifts for the ethylene trithiocarbonate complex with iodine in carbon disulphide.

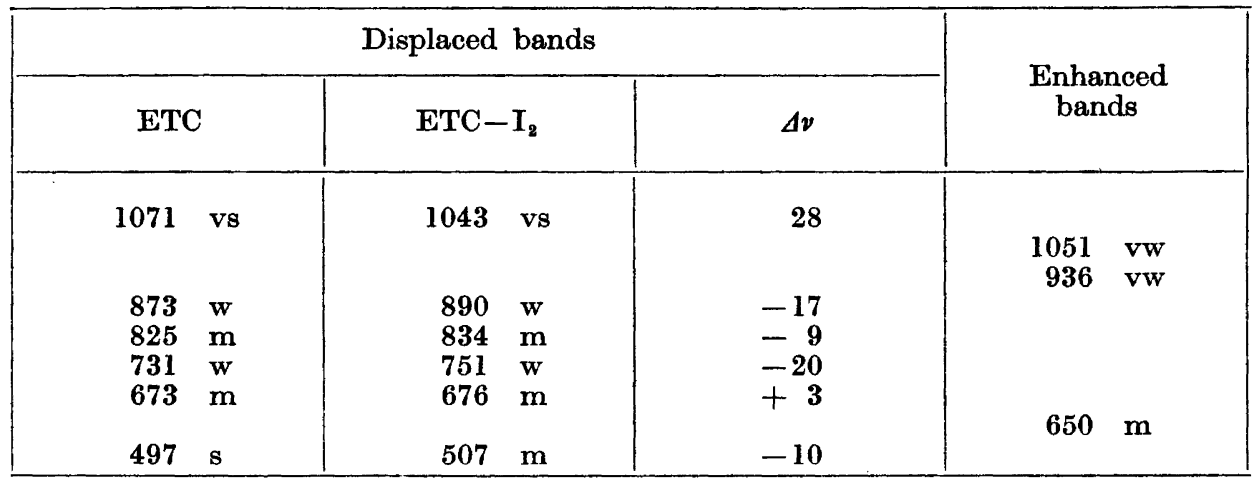

For abbreviations, see Table 1.

ETC in $\mathrm{CHCl}_{3}$, iodine was added to a $0.1 \mathrm{M}$ concentration, which was the maximum that would permit a Raman spectrum to be recorded with the $6328 \AA$ excitation. A small peak was observed at $1040 \mathrm{~cm}^{-1}$, the shift corresponding to the infrared results. However, the low intensity of the peak would suggest that the $C=S$ stretching mode in the complex is weaker than for the ETC itself. To our knowledge this is the first time spectral shifts of the donor on complexing with iodine has ever been reported in Raman, and attempts ${ }^{13}$ with other donors like dimethyl sulphoxide, diethyl sulphide and pyridine $\mathrm{N}$-oxide were negative.

\section{Ethylene thiourea}

ETU is only slightly soluble in organic solvents and this molecule could therefore only be studied in the solid state. The point group for ETU is probably $C_{s}$, since the ring appears planar, but the $S$ atom is lying outside this plane. ${ }^{11}$ Our vibrational spectra suggest that the point group cannot be $C_{2 v}$, but since no polarization data were available, any distinction between $C_{s}$, $C_{2}$, or $C_{1}$ in the crystal is not possible.

The tentative vibrational assignments are listed in Table 3 and only a few comments will be made. Since ETU contains 12 atoms, there are 30 fundamentals. In addition to those encountered in ETC there are two $\mathrm{N}-\mathrm{H}$ stretching modes around $3400 \mathrm{~cm}^{-1}$, two $\mathrm{N}-\mathrm{H}$ in plane (around $1500 \mathrm{~cm}^{-1}$ ) and two $\mathrm{N}-\mathrm{H}$ out of plane deformation modes (around $700 \mathrm{~cm}^{-1}$ ). The assigned frequencies agree reasonably well with those found for ETC and again there are surprisingly many low frequencies, indicating a "soft" flexible framework. The $\mathrm{C}=\mathrm{S}$ stretching mode has been assigned to the bands at $1208 \mathrm{~cm}^{-1}$ in agreement with Mecke et al. ${ }^{1}$ It is well known that for $\mathrm{C}=\mathrm{S}$ groups geminal to an $\mathrm{N}$-atom strong coupling with the $\mathrm{N}-\mathrm{H}$ deformation modes occur. ${ }^{9}$ The very strong band at $506-508 \mathrm{~cm}^{-1}$ is assigned to the ring breathing mode,

Acta Chem. Scand. 22 (1968) No. 5 
very close to the corresponding band in ETC. The strong, very low Raman band at $86 \mathrm{~cm}^{-1}$ cannot be a fundamental and is assigned as a lattice mode.

It is well known that thiourea itself forms strong charge transfer complexes with iodine. ${ }^{20}$ A qualitative study of the ETU $-I_{2}$ complex dissolved in $\mathrm{CH}_{2} \mathrm{Cl}_{2}$ was carried out in the ultraviolet and visible regions. The curves were very similar to those reported for thiourea. ${ }^{20}$ No infrared study of the ETU $-\mathrm{I}_{2}$ complex could be carried out because of the low solubility. Such studies would have been of interest for ETU and might have given more information about the vibrational coupling of the $\mathrm{C}=\mathrm{S}$ stretching mode.

The author is grateful to Professor Ellis R. Lippincott of the University of Maryland for working facilities and to the Applied Physics Corporation for extended use of the Cary 81 laser Raman spectrometer. Financial aid form Guldborg and Waage's fund is grat-fully acknowledged.

\section{REFERENCES}

1. Mecke. R. and Mrcke. R. (Hicm. Ber. 89 (1956) 343: Mecke. R., Mecke, R. and Lüttringhaus, A. Chem. Ber. 90 (1957) 975.

2. Angell, C. L. Trans. Faraday Soc. 52 (1956) 1178.

3. Katon, J. E. and Feairheller, W. R. Spectrochim. Acta 21 (1965) 2011.

4. Klæboe, P. Acta Chemn. Scand. 22 (1968) 369.

5. Yamaguchi, A., Penland, R. B., Mizushima, S., Lane, T. J., Curran, C. and Quagliano, J. V. J. Am. Chem. Soc. 80 (1958) 527.

6. Kutzelnigg, W. and Mecke, R. Spectrochim. Acta 17 (1961) 530.

7. Spinner, E. Spectrochim. Acta 15 (1959) 95.

8. Bellamy, L. J. and Rogasch, P. E. J. Chem. Soc. 19602218.

9. Elmore, D. T. J. Chem. Soc. 19583489.

10. Rao, C. N. R. and Venkataraghavan, R. Spectrochim. Acta 17 (1962) 541.

11. Wheatley, P. J. Acta Cryst. 6 (1953) 369.

12. Brown, C. J. Acta Cryst. 7 (1954) 92.

13. Klæboe, P. J. Am. Chem. Soc. 89 (1967) 3667.

14. Rico, M., Orza, J. M. and Morcillo, J. Spectrochim. Acta 22 (1966) 1417.

15. Morcillo, J. and Orza, J. M. Anales Real Soc. Espan. Fis. Quim. (Madrid) B 56 (1960) 231.

16. Christensen, D. H., Nielsen, J. T. and Nielsen, O. F. J. Mol. Spectry. 24 (1967) 225, 477; 25 (1968) 197.

17. Briegleb, G. Elektronen-Donator-Acceptor-Komplexe, Springer, Berlin 1961, p. 79.

18. Bhaskar, K. R., Bhat, S. N., Murthy, A. S. N. and Rao, C. N. R. Trans. Faraday Soc. 62 (1966) 788.

19. Augdahl, E. and Klæboe, P. Acta Chem. Scand. 16 (1962) 1637.

20. Lang, R. P. J. Am. Chem. Soc. 84 (1962) 1185.

Received November 22, 1967. 